\title{
Near-Field to Far-Field Transformation Techniques with Spiral Scannings: A Comprehensive Review
}

\author{
Renato Cicchetti, ${ }^{1}$ Francesco D'Agostino, ${ }^{2}$ Flaminio Ferrara, \\ Claudio Gennarelli, ${ }^{2}$ Rocco Guerriero, ${ }^{2}$ and Massimo Migliozzi ${ }^{2}$ \\ ${ }^{1}$ Dipartimento di Ingegneria dell'Informazione, Elettronica e Telecomunicazioni, Università di Roma "La Sapienza", \\ Via Eudossiana 18, 00184 Roma, Italy \\ ${ }^{2}$ Dipartimento di Ingegneria Industriale, Università di Salerno, Via Giovanni Paolo II, 132-84084 Fisciano, Italy \\ Correspondence should be addressed to Claudio Gennarelli; gennar@diiie.unisa.it
}

Received 12 June 2014; Accepted 16 July 2014; Published 10 August 2014

Academic Editor: Diego Caratelli

Copyright (C) 2014 Renato Cicchetti et al. This is an open access article distributed under the Creative Commons Attribution License, which permits unrestricted use, distribution, and reproduction in any medium, provided the original work is properly cited.

\begin{abstract}
An overview of the near-field-far-field (NF-FF) transformation techniques with innovative spiral scannings, useful to derive the radiation patterns of the antennas commonly employed in the modern wireless communication systems, is provided in this paper. The theoretical background and the development of a unified theory of the spiral scannings for quasi-spherical and nonspherical antennas are described, and an optimal sampling interpolation expansion to evaluate the probe response on a quite arbitrary rotational surface from a nonredundant number of its samples, collected along a proper spiral wrapping it, is presented. This unified theory can be applied to spirals wrapping the conventional scanning surfaces and makes it possible to accurately reconstruct the NF data required by the NF-FF transformation employing the corresponding classical scanning. A remarkable reduction of the measurement time is so achieved, due to the use of continuous and synchronized movements of the positioning systems and to the reduced number of needed NF measurements. Some numerical and experimental results relevant to the spherical spiral scanning case when dealing with quasi-planar and electrically long antennas are shown.
\end{abstract}

\section{Introduction}

The design of the modern antenna systems, integrated on portable devices or employed in radio base stations, requires the experimental verification of the antenna performances in terms of frequency bands and radiation diagrams. Different types of antennas are employed in the abovementioned applications. In particular, printed or conformal antennas are generally used in portable devices (such as cellular phones, mobile pc, printers, scanners, DVD players, and digital projectors) [1-6], collinear or dielectric resonator antennas are adopted to realize broadcast and cellular base stations [7-14], while reflector or horn antennas are usually adopted for radio links [15-17]. Consequently, different types of measurement techniques are required to measure the corresponding antenna radiation patterns.

As well known, the accurate measurement of the electromagnetic (EM) field radiated by an antenna can be performed only in an anechoic chamber, wherein the freespace propagation conditions are emulated by suppressing a large amount of the reflections from the lateral walls, ceiling, and floor. However, the far-field (FF) distance requirements cannot be practically satisfied in an anechoic chamber when dealing with antennas having large or even medium electrical dimensions, so that only near-field (NF) measurements can be carried out. This occurs specifically when characterizing antenna systems and arrays for space as well as radar applications $[18,19]$. Therefore, the NF-FF transformation techniques, which allow an accurate reconstruction of the antenna far-field pattern from measurements in the NF region, have been widely investigated [20-28] and employed in the last forty years as a viable and more accurate alternative to the direct FF measurements performed on a conventional FF range. In addition, NF scanning techniques represent the better choice when complete pattern and polarization measurements are required. Moreover, they provide the necessary 
information for determining the field at the surface of the antenna and such information can be properly employed for the diagnostics of surface errors in a reflector antenna or of faulty elements in an array (microwave holographic diagnostics [29]).

Usually, the acquired NF data are transformed into FF patterns by using a suitable expansion of the field of the antenna under test (AUT) in terms of modes, that is, a complete set of solutions of the vector wave equation in the region outside the antenna. To this end, plane, cylindrical, or spherical waves are generally employed [20-27]. The modal expansion adopted to represent the spatial field distribution determines the type of the NF scanning surface, which accordingly will be a plane, a cylinder, or a sphere. The orthogonality properties of the modes on these surfaces are then properly exploited to determine the modal expansion coefficients, whose knowledge allows the reconstruction of the AUT far field. The development and the spreading of NF-FF transformation techniques with planar, cylindrical, or spherical scanning are justified from the fact that each technique has its own particular advantages and limitations.

In this framework, the application of the spatial bandlimitation properties of radiated EM fields [30] and of their nonredundant sampling representations $[31,32]$ to the voltage measured by the scanning probe has allowed the development of effective nonredundant probe-compensated NF-FF transformation techniques. The interested reader can refer to $[32,33]$ for an extensive bibliography. These techniques require typically a number of NF data remarkably smaller than that needed when employing the corresponding classical ones. As a matter of fact, the NF data needed by these last ones are accurately reconstructed from the acquired nonredundant data by means of optimal sampling interpolation (OSI) expansions. It is so possible to obtain a remarkable measurement time saving and this is a very important result, since nowadays such a time is very much greater than the computational one required to carry out the NF-FF transformation.

The time needed for the acquisition of the NF data can be drastically reduced by using the modulated scattering technique, wherein arrays of scattering probes, which allow a very fast electronic scanning, are employed [34]. However, apart from measurement precision issues, antenna NF measurement systems adopting such a technique are not very flexible. In any case, the number of needed probes could be significantly reduced by properly exploiting the nonredundant sampling representations.

A more convenient way for reducing the measurement time is, as suggested in [35], the employment of spiral scanning techniques, which can be accomplished by means of continuous and synchronized movements of the positioning systems of the AUT and probe. Accurate, stable, and effective, nonredundant NF-FF transformations with helicoidal scanning [33, 36-42], planar [37, 42-44], and spherical [37, 42, 45-52] spiral scannings (Figures 1, 2, and 3) have been developed in the last years. They are based on the nonredundant sampling representations and recover the NF data needed by the standard NF-FF transformation, which employs the corresponding classical scanning (cylindrical [53], planerectangular [54], and spherical [55]), by interpolating, via proper OSI expansions, the nonredundant samples acquired on the spiral. The nonredundant representations and the related OSI expansions have been obtained in [36-38, 43, $45,46]$ by assuming the AUT as enclosed in the smallest sphere containing it, whereas more effective AUT modellings, allowing a further reduction of needed NF samples when considering nonspherical antennas (i.e., antennas with one or two predominant dimensions), have been used in [39$42,44,47-52]$. In all the cases, they have been determined:

(1) by choosing the spiral in such a way that its step, specified by two consecutive intersections with the considered meridian curve (generatrix, radial line, and meridian), be equal to the sample spacing needed for the interpolation along this curve;

(2) by developing the nonredundant sampling representation along the spiral.

It must be stressed that the use of effective AUT modellings when dealing with elongated or quasi-planar antennas offers, besides the reduction of the number of required NF data, another great advantage in the helicoidal and planar spiral scanning case, respectively. Specifically, it makes possible the consideration of measurement cylinders (planes) with a radius (distance) smaller than one-half of the antenna maximum size, thus reducing the error related to the truncation of the scanning surface.

A unified theory of the spiral scannings for spherical and nonspherical antennas has been also developed in [37] and $[33,42]$, respectively.

Moreover, direct NF-FF transformations with helicoidal scanning, which allow the evaluation of the antenna far field in any cut plane directly from a minimum set of NF data without interpolating them, have been recently proposed in [56-58].

Other NF-FF transformation techniques with spiral scannings have been also proposed $[35,59,60]$. However, since they do not take advantage of the nonredundant sampling representations of EM fields, they require a useless large amount of NF measurements and, as a consequence, a greater acquisition time.

The aim of this paper is just to provide an overview of the NF-FF transformation techniques with spiral scannings, useful to characterize the antennas commonly used in the modern wireless communication systems. The necessary theoretical background on the nonredundant sampling representations of EM fields is summarized in Section 2. In Section 3, the development of a unified theory of the NFFF transformations with spiral scannings for spherical or nonspherical antennas is described, and a two-dimensional OSI expansion, which allows reconstructing the probe voltage on a quite arbitrary rotational surface from its nonredundant samples collected along a proper spiral wrapping it, is presented, while some representative numerical and experimental results relevant to the spherical spiral scanning case when dealing with quasi-planar and electrically long antennas, respectively, are reported in Section 4. Finally, the conclusions are drawn in Section 5. 


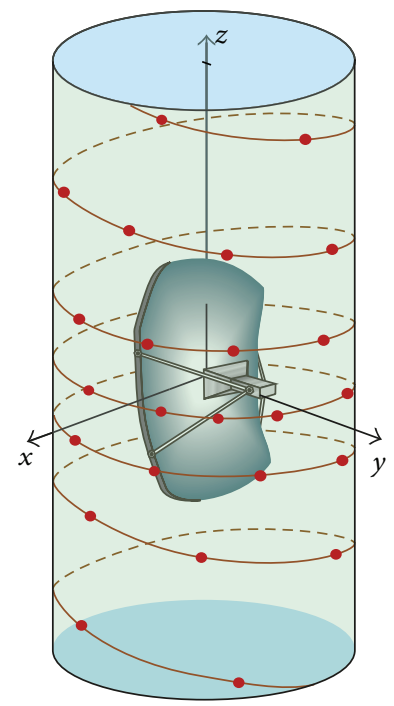

Figure 1: Helicoidal scanning.

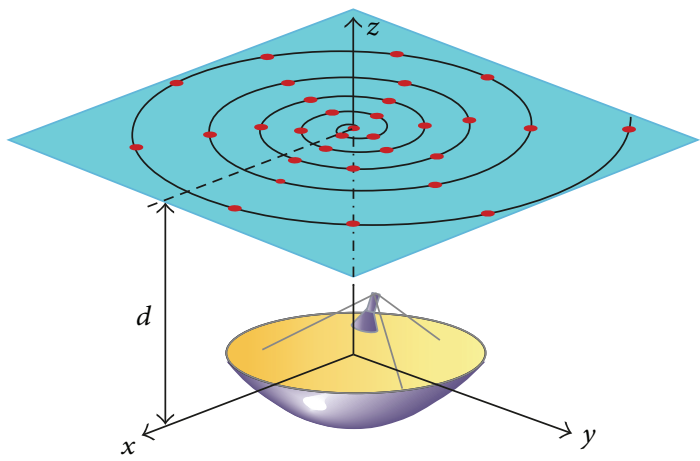

FIGURE 2: Planar spiral scanning.

\section{Theoretical Background}

Let us consider an antenna enclosed in a convex domain $\mathscr{D}$ of finite size, bounded by a surface $\Sigma$ with rotational symmetry and a nondirective probe scanning an arbitrary surface $\mathscr{M}$, external to $\mathscr{D}$ and having the same rotational symmetry. Since the voltage $V$ measured by such a probe has the same effective spatial bandwidth of the field radiated by the AUT [61], the nonredundant sampling representations of EM fields [31] can be applied to it. Accordingly, it is convenient to introduce the "reduced voltage":

$$
\widetilde{V}(\xi)=V(\xi) e^{j \gamma(\xi)}
$$

where $V(\xi)$ is the voltage measured by the probe, $\gamma(\xi)$ is a proper phase function, and $\underline{r}=\underline{r}(\xi)$ is the optimal parameterization adopted to describe the observation curve $C$ on the surface $\mathscr{M}$. The bandlimitation error, occurring when $\widetilde{V}(\xi)$ is approximated by a bandlimited function, exhibits a

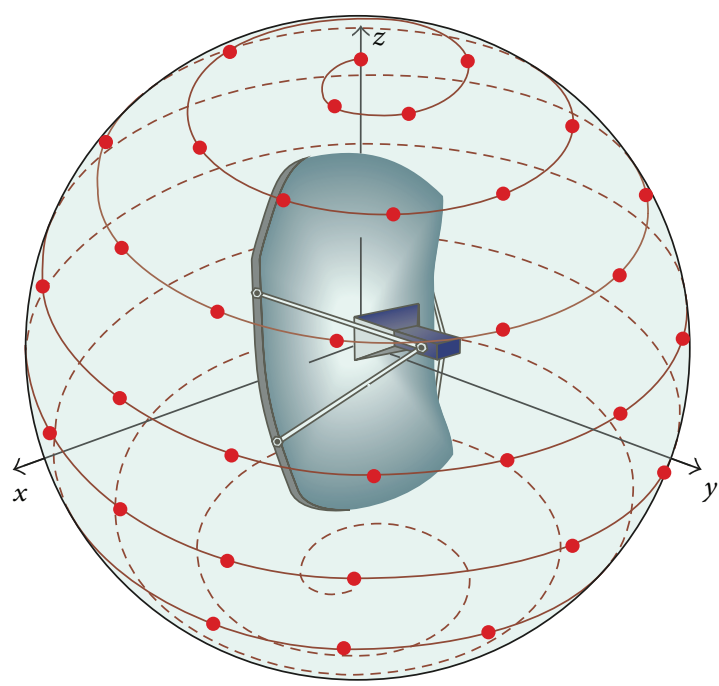

FIGURE 3: Spherical spiral scanning.

step-like behaviour and becomes negligible [31] as the spatial bandwidth exceeds the critical value:

$$
W_{\xi}=\max _{\xi}[w(\xi)]=\max _{\xi}\left[\max _{\underline{r}^{\prime}}\left|\frac{\mathrm{d} \gamma(\xi)}{\mathrm{d} \xi}-\beta \frac{\partial R\left(\xi, \underline{r}^{\prime}\right)}{\partial \xi}\right|\right],
$$

where $\beta$ is the free-space wavenumber, $r^{\prime}$ denotes the source point, and $R=\left|\underline{r}(\xi)-\underline{r}^{\prime}\right|$. Accordingly, such an error can be effectively controlled by choosing the bandwidth of the approximating function equal to $\chi^{\prime} W_{\xi}$, where $\chi^{\prime}$ is an enlargement bandwidth factor (slightly greater than unity for electrically large antennas).

To obtain a nonredundant representation, that is, a representation requiring a minimum number of samples, first of all, we must minimize the "local" bandwidth $w(\xi)$ for each $\xi$. This is obtained by choosing $\gamma$ such that its derivative is the average between the maximum and minimum value of $\beta \partial R / \partial \xi$, when $\underline{r}^{\prime}$ varies in $\mathscr{D}$; that is,

$$
\begin{aligned}
\frac{\mathrm{d} \gamma}{\mathrm{d} \xi} & =\frac{\beta}{2}\left[\max _{\underline{r}^{\prime}} \frac{\partial R}{\partial s}+\min _{\underline{r}^{\prime}} \frac{\partial R}{\partial s}\right] \frac{\mathrm{d} s}{\mathrm{~d} \xi} \\
& =\frac{\beta}{2}\left[\max _{\underline{r}^{\prime}} \widehat{R} \cdot \hat{t}+\min _{\underline{r}^{\prime}} \widehat{R} \cdot \hat{t}\right] \frac{\mathrm{d} s}{\mathrm{~d} \xi},
\end{aligned}
$$

where $s$ is the curvilinear abscissa along $C, \widehat{R}$ is the unit vector pointing from the source point to the observation point $P$, and $\hat{t}$ is the unit vector tangent to $C$ at $P$. Accordingly, the optimal phase factor to be used is given by

$$
\begin{aligned}
\gamma(\xi) & =\frac{\beta}{2} \int_{0}^{s(\xi)}\left[\max _{\underline{r}^{\prime}} \frac{\partial R}{\partial s}+\min _{\underline{r}^{\prime}} \frac{\partial R}{\partial s}\right] \mathrm{d} s \\
& =\frac{\beta}{2} \int_{0}^{s}\left[\max _{\underline{r}^{\prime}} \widehat{R} \cdot \hat{t}+\min _{\underline{r}^{\prime}} \widehat{R} \cdot \hat{t}\right] \mathrm{d} s .
\end{aligned}
$$


It can be easily recognized that, by choosing $\mathrm{d} \gamma / \mathrm{d} \xi$ according to (3), the following is obtained:

$$
\begin{aligned}
w(\xi) & =\frac{\beta}{2}\left[\max _{\underline{r}^{\prime}} \frac{\partial R}{\partial s}-\min _{\underline{r}^{\prime}} \frac{\partial R}{\partial s}\right] \frac{\mathrm{d} s}{\mathrm{~d} \xi} \\
& =\frac{\beta}{2}\left[\max _{\underline{r}^{\prime}} \widehat{R} \cdot \widehat{t}-\min _{\underline{r}^{\prime}} \widehat{R} \cdot \widehat{t}\right] \frac{\mathrm{d} s}{\mathrm{~d} \xi} .
\end{aligned}
$$

As regards the parameter $\xi$, in order to avoid redundancy, it must be determined by requiring that the local bandwidth is constant. In fact, if $w(\xi)$ is variable with $\xi$, the sample spacing, which is fixed by the bandwidth $W_{\xi}$, becomes unnecessarily small in the zones wherein $w(\xi)$ is smaller than its maximum value, thus giving rise to redundancy in the sampling representation. Accordingly, by imposing that $w(\xi)$ is constant and equal to $W_{\xi}$, we obtain

$$
\begin{aligned}
\xi & =\frac{\beta}{2 W_{\xi}} \int_{0}^{s}\left[\max _{\underline{r}^{\prime}} \frac{\partial R}{\partial s}-\min _{\underline{r}^{\prime}} \frac{\partial R}{\partial s}\right] \mathrm{d} s \\
& =\frac{\beta}{2 W_{\xi}} \int_{0}^{s}\left[\max _{\underline{r}^{\prime}} \widehat{R} \cdot \widehat{t}-\min _{\underline{r}^{\prime}} \widehat{R} \cdot \widehat{t}\right] \mathrm{d} s .
\end{aligned}
$$

It can be easily verified that when $C$ is a meridian curve (i.e., a curve lying on a plane at $\varphi=$ const, $\varphi$ being the usual azimuthal angle of the spherical coordinate system $(r, \vartheta, \varphi)$ ) and $\hat{t}$ is external to the cone of vertex $P$, tangent to $\Sigma$, the extreme values of the inner product $\widehat{R} \cdot \hat{t}$ occur (Figure 4 ) at the two tangency points $P_{1,2}$ on $C^{\prime}$ (intersection curve between the meridian plane and $\Sigma$ ). By taking into account that in such a case [31]

$$
\left.\frac{\partial R}{\partial s}\right|_{s_{1,2}^{\prime}}=\frac{\mathrm{d} R_{1,2}}{\mathrm{~d} s} \pm \frac{\mathrm{d} s_{1,2}^{\prime}}{\mathrm{d} s},
$$

where $s_{1,2}^{\prime}$ are the arclength coordinates of $P_{1,2}$ and $R_{1,2}$ the distances from $P$ to $P_{1,2}$ (Figure 4 ), and choosing $W_{\xi}=$ $\beta \ell^{\prime} / 2 \pi$ ( $\ell^{\prime}$ being the length of $C^{\prime}$ ), the following is obtained:

$$
\begin{aligned}
& \gamma=\frac{\beta}{2}\left[R_{1}+R_{2}+s_{1}^{\prime}-s_{2}^{\prime}\right], \\
& \xi=\frac{\pi}{\ell^{\prime}}\left[R_{1}-R_{2}+s_{1}^{\prime}+s_{2}^{\prime}\right] .
\end{aligned}
$$

Note that the angular-like parameter $\xi$ covers a $2 \pi$ range, when $P$ encircles the antenna once, and the number of samples at Nyquist spacing $\left(\Delta \xi=\pi / W_{\xi}\right)$ on a closed meridian curve $C$ (also unbounded) is always finite and equal to $N_{\xi}=$ $2 \pi / \Delta \xi=2 \ell^{\prime} / \lambda, \lambda$ being the free-space wavelength.

It can be shown [31] that, in any meridian plane, the curves $\gamma=$ const and $\xi=$ const are orthogonal.

When $\Sigma$ is chosen coincident with a sphere of radius $a$, we have

$$
\begin{gathered}
R_{1}=R_{2}=\sqrt{r^{2}-a^{2}}, \\
s_{1}^{\prime}=(\theta-\alpha) a ; \quad s_{2}^{\prime}=(\theta+\alpha) a ; \quad \alpha=\cos ^{-1}\left(\frac{a}{r}\right) .
\end{gathered}
$$

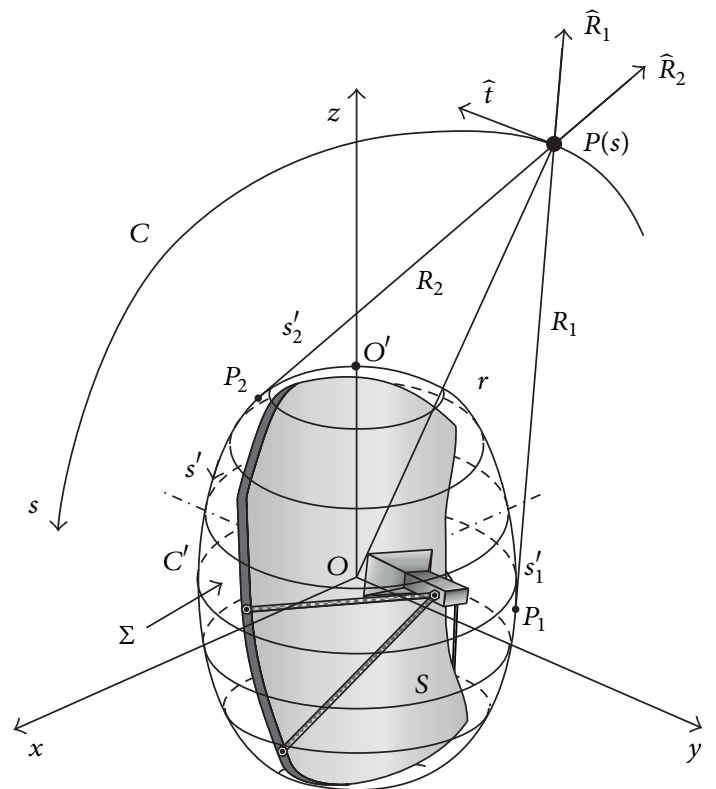

Figure 4: Relevant to a meridian observation curve.

Accordingly, since $\ell^{\prime}=2 \pi a$, it results $W_{\xi}=\beta a$ and

$$
\gamma=\beta \sqrt{r^{2}-a^{2}}-\beta a \cos ^{-1}\left(\frac{a}{r}\right) ; \quad \xi=\theta,
$$

wherein the parameter $\theta$ coincides in the range $[0, \pi]$ with the zenithal angle $\vartheta$.

It must be stressed that, according to [31], the surface $\Sigma$ modelling the AUT must fit very well its shape in order to minimize the overall number of needed samples. As a matter of fact, the number of samples at Nyquist spacing on any closed observation surface (also unbounded) surrounding the antenna is

$$
N \cong \frac{\text { area of } \Sigma}{(\lambda / 2)^{2}}
$$

As a consequence, an effective source modelling when dealing with an electrically long antenna is got, f.i., by choosing $\Sigma$ coincident with a prolate ellipsoid with major and minor semiaxes equal to $a$ and $b$ (Figure 5), whereas a quasi-planar antenna can be conveniently modelled by an oblate ellipsoid. In both cases, it can be shown [31] that, in any meridian plane, the curves $\gamma=$ const and $\xi=$ const are ellipses and hyperbolas confocal to $C^{\prime}$ (see Figure 5). Accordingly, $\xi$ and $\gamma$ are functions of the elliptic coordinates $u=\left(r_{1}-r_{2}\right) / 2 f$ and $v=\left(r_{1}+r_{2}\right) / 2 a$, respectively, where $r_{1,2}$ are the distances from the observation point $P$ to the foci of $C^{\prime}$ and $2 f$ is the focal distance. By taking into account that the length of the ellipse $C^{\prime}$ is $\ell^{\prime}=4 a \mathrm{E}\left(\pi / 2 \mid \varepsilon^{2}\right)$, the bandwidth $W_{\xi}$ becomes

$$
W_{\xi}=\left(\frac{4 a}{\lambda}\right) \mathrm{E}\left(\frac{\pi}{2} \mid \varepsilon^{2}\right),
$$

wherein $\mathrm{E}(\cdot \mid \cdot)$ denotes the elliptic integral of second kind and $\varepsilon=f / a$ is the eccentricity of $C^{\prime}$. By straightforward 


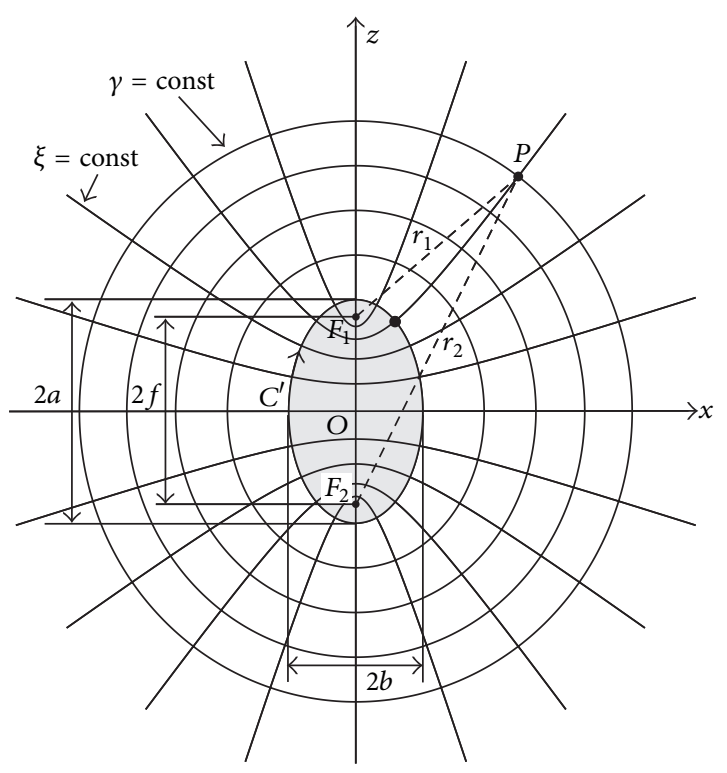

FIGURE 5: Ellipsoidal source modelling: prolate case.

but lengthy computations, from (8), the following is obtained [31]:

$$
\begin{gathered}
\gamma=\beta a\left[v \sqrt{\left.\frac{v^{2}-1}{v^{2}-\varepsilon^{2}}-\mathrm{E}\left(\cos ^{-1} \sqrt{\frac{1-\varepsilon^{2}}{v^{2}-\varepsilon^{2}}} \mid \varepsilon^{2}\right)\right],}\right. \\
\xi=\frac{\pi}{2} \begin{cases}\frac{\mathrm{E}\left(\sin ^{-1} u \mid \varepsilon^{2}\right)}{\mathrm{E}\left(\pi / 2 \mid \varepsilon^{2}\right)}+1 & \text { prolate ellipsoid } \\
\frac{\mathrm{E}\left(\sin ^{-1} u \mid \varepsilon^{2}\right)}{\mathrm{E}\left(\pi / 2 \mid \varepsilon^{2}\right)} & \text { oblate ellipsoid. }\end{cases}
\end{gathered}
$$

Relation (14) is valid when the angle $\vartheta$ corresponding to $P$ belongs to the range $[0, \pi / 2]$. The case in which $\vartheta$ belongs to $[\pi / 2, \pi]$ can be easily handled by determining the value $\xi^{\prime}$ corresponding to the point specified by $\pi-\vartheta$ and then putting $\xi=\pi-\xi^{\prime}$.

It can be easily shown [31] that the spherical modelling can be obtained from the prolate or oblate ellipsoidal one by considering an ellipsoid with eccentricity $\varepsilon=0$. It is worth noting that in such a case the curves $\gamma=$ const and $\xi=$ const become circumferences and radial lines, respectively.

\section{Nonredundant Spiral Scanning Techniques}

Let us now consider a nondirective probe scanning a proper spiral wrapping an arbitrary rotational surface $\mathscr{M}$ obtained by rotating a meridian curve always external to the cone having the vertex at $P$ and tangent to the rotational surface $\Sigma$ enclosing the AUT. The parametric equations of such a spiral are

$$
\begin{aligned}
& x=r(\theta) \sin \theta \cos \phi, \\
& y=r(\theta) \sin \theta \sin \phi, \\
& z=r(\theta) \cos \theta,
\end{aligned}
$$

wherein $\phi$ is the angular parameter describing the spiral, $r(\theta)$ is specified by the meridian curve generating the surface $\mathscr{M}$, and the angle $\theta$ is a monotonic increasing function of $\phi$. It can be easily recognized that $r(\theta)=d / \cos \theta$ in the case of a planar spiral lying on a plane at distance $d$ and $r(\theta)=d / \sin \theta$ for a helix wrapping a cylinder of radius $d$, whereas $r(\theta)=d$ when a spherical spiral is considered. It must be stressed that the spiral angle $\theta$, unlike the zenithal one $\vartheta$, can take negative values. In fact, when the spiral describes a complete round on the surface $\mathscr{M}$, moving from the south pole to the north pole and then returning to the south one, $\theta$ varies in the range $[-\pi, \pi]$. Moreover, the angular parameter $\phi$ is always continuous, whereas, according to (15), the azimuthal angle $\varphi$ exhibits a discontinuity jump of $\pi$ when the spiral crosses the poles.

In order to obtain a nonredundant sampling representation of the probe voltage on the surface $\mathscr{M}$ from its samples acquired on the spiral, it is necessary [42]

(1) to choose the spiral in such a way that its pitch, specified by two consecutive intersections with a meridian curve, be equal to the sample spacing needed for the interpolation along this curve,

(2) to develop a nonredundant sampling representation along the spiral.

According to condition (1), the optimal parameter $\xi$ relevant to the meridian curve and the angular one $\phi$ describing the spiral are related by

$$
\xi=k \phi
$$

where $k$ must be such that the interval $\Delta \xi=2 \pi k$, corresponding to the spiral pitch, is equal to the required [31] sample spacing $2 \pi /\left(2 N^{\prime \prime}+1\right)$, with $N^{\prime \prime}=\operatorname{Int}\left(\chi N^{\prime}\right)+1$ and $N^{\prime}=$ $\operatorname{Int}\left(\chi^{\prime} W_{\xi}\right)+1, \operatorname{Int}(x)$ denoting the integer part of $x$ and $\chi>1$ being an oversampling factor. Accordingly, $k=1 /\left(2 N^{\prime \prime}+1\right)$.

The development of a nonredundant sampling representation of the voltage along the spiral is a more difficult task, which has been heuristically tackled in [42] by paralleling the rigorous procedure [37] valid when adopting a spherical AUT modelling.

Accordingly, the main results of the unified theory of spiral scannings for antennas enclosed in a spherical surface [37] are recalled in the following. In such a case, the optimal parameter for describing a meridian curve is $\theta$ and the related bandwidth is $\beta a$. Moreover, the optimal phase factor $\psi$ and parameter $\eta$ to get a nonredundant representation along the spiral can be obtained from (4) and (6) by taking into account that the extreme values of $\widehat{R} \cdot \hat{t}$ occur [37] at the two tangency points $P_{1,2}$ (Figure 6) between the sphere modelling the AUT and the straight lines passing through the point $Q$ on the spiral and belonging to the plane specified by the unit vectors $\widehat{t}$ (tangent to the spiral at $Q$ ) and $\widehat{r}$ (pointing from the origin to the point $Q$ ). Denoting by $\widehat{R}_{1,2}$ the related unit vectors and 
by $\delta$ the angle between $\hat{r}$ and $\hat{t}$ (Figure 6), it can be easily verified that [37]

$$
\begin{gathered}
\frac{\left(\widehat{R}_{1}+\widehat{R}_{2}\right)}{2}=\widehat{r} \sin \alpha=\widehat{r} \sqrt{1-\frac{a^{2}}{r^{2}}}, \\
\frac{\left(\widehat{R}_{1}-\widehat{R}_{2}\right)}{2}=\widehat{n} \cos \alpha,
\end{gathered}
$$

where $\widehat{n}$ is a unit vector orthogonal to $\widehat{r}$. Therefore

$$
\frac{\left(\widehat{R}_{1}-\widehat{R}_{2}\right) \cdot \widehat{t}}{2}=(\widehat{n} \cdot \widehat{t})\left(\frac{a}{r}\right)=\left(\frac{a}{r}\right) \sin \delta .
$$

By substituting (17) in (4) and taking into account that $\mathrm{d} r=\widehat{r} \cdot \widehat{t} \mathrm{~d} s$, the following is obtained:

$$
\psi=\beta \int_{0}^{r} \sqrt{1-\frac{a^{2}}{r^{2}}} \mathrm{~d} r=\beta \sqrt{r^{2}-a^{2}}-\beta a \cos ^{-1}\left(\frac{a}{r}\right)
$$

that is, when adopting a spherical modelling, the phase function $\psi$ relevant to the sampling representation along the spiral coincides with that $\gamma$ relevant to the representation on a meridian curve.

On the other hand [37],

$$
\mathrm{d} s=\sqrt{r^{2} \sin ^{2} \theta+k^{2} r^{2}+k^{2} \dot{r}^{2}} \mathrm{~d} \phi,
$$

wherein $\dot{r}=\mathrm{d} r / \mathrm{d} \theta$. Moreover,

$$
\begin{aligned}
\widehat{r} \cdot \hat{t} & =\frac{\mathrm{d} r}{\mathrm{~d} s}=\frac{\mathrm{d} r}{\mathrm{~d} \phi} \frac{\mathrm{d} \phi}{\mathrm{d} s}=\left[\frac{\mathrm{d} r}{\mathrm{~d} \theta} \frac{\mathrm{d} \theta}{\mathrm{d} \phi}\right] \frac{\mathrm{d} \phi}{\mathrm{d} s}=k \dot{r} \frac{\mathrm{d} \phi}{\mathrm{d} s} \\
& =\frac{k \dot{r}}{\sqrt{r^{2} \sin ^{2} \theta+k^{2} r^{2}+k^{2} \dot{r}^{2}}}
\end{aligned}
$$

and, therefore,

$$
\sin \delta=\sqrt{1-(\widehat{r} \cdot \widehat{t})^{2}}=\sqrt{\frac{r^{2} \sin ^{2} \theta+k^{2} r^{2}}{r^{2} \sin ^{2} \theta+k^{2} r^{2}+k^{2} \dot{r}^{2}}} .
$$

By taking into account such a relation and substituting (19) and (21) in (6), we get

$$
\eta=\frac{\beta a}{W_{\eta}} \int_{0}^{\phi} \sqrt{k^{2}+\sin ^{2} k \phi} \mathrm{d} \phi
$$

According to such a relation, when adopting a spherical modelling, the optimal parameter $\eta$ for describing the spiral is $\beta / W_{\eta}$ times the arclength of the projecting point that lies on the spiral wrapping the sphere $\Sigma$.

It is worth noting that the scanning spiral can be viewed as obtained by radially projecting the corresponding one wrapping, with the same pitch, the modelling sphere. Since such a spiral is a closed curve, it is convenient to choose the bandwidth $W_{\eta}$ in such a way that the angular-like parameter $\eta$ covers a $2 \pi$ range when the whole projecting curve is described. Accordingly,

$$
W_{\eta}=\frac{\beta a}{\pi} \int_{0}^{\left(2 N^{\prime \prime}+1\right) \pi} \sqrt{k^{2}+\sin ^{2} k \phi} \mathrm{d} \phi ;
$$

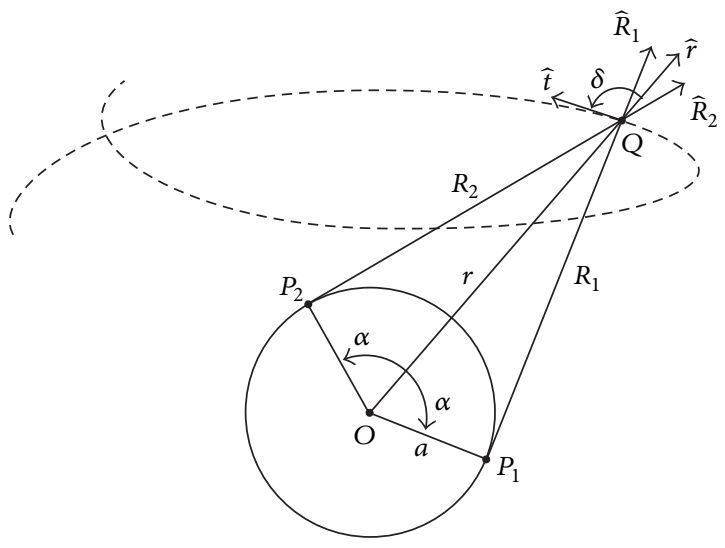

FIGURE 6: Geometry of the problem in the plane $\widehat{t}, \widehat{r}$.

that is, the bandwidth $W_{\eta}$ is $\beta / \pi$ times the length of the spiral which wraps from pole to pole the modelling sphere.

Let us now turn to the more general case wherein the AUT is no longer modelled as enclosed in a sphere. The parameterization $\eta$ to describe the scanning spiral, the related phase factor $\psi$, and bandwidth $W_{\eta}$ can be heuristically determined [42] by generalizing the corresponding relations for the case of the spherical modelling (see (24), (20), and (25)). In particular, $\eta$ is $\beta / W_{\eta}$ times the arclength of the projecting point that lies on the spiral wrapping the surface $\Sigma$ (the projection is obtained by the curves at $\xi=$ const that, in such a case, take the role of the radial lines of the spherical modelling), $\psi$ coincides with the phase function $\gamma$ relevant to a meridian curve, and the bandwidth $W_{\eta}$ is $\beta / \pi$ times the length of the spiral wrapping $\Sigma$ from pole to pole. In other words, the spiral, $\psi$, and $\eta$ are such that they coincide with those relevant to the spherical modelling when the surface $\Sigma$ approaches a sphere.

According to these results, the reduced voltage at any point $Q$ of the spiral can be reconstructed by the following OSI expansion [37, 42]:

$$
\widetilde{V}(\eta)=\sum_{m=m_{0}-p+1}^{m_{0}+p} \widetilde{V}\left(\eta_{m}\right) \Omega_{M}\left(\eta-\eta_{m}\right) D_{M^{\prime \prime}}\left(\eta-\eta_{m}\right),
$$

where $2 p$ is the number of retained samples $\widetilde{V}\left(\eta_{m}\right), m_{0}=$ $\operatorname{Int}(\eta / \Delta \eta)$ is the index of the sample nearest (on the left) to the point $Q$, and

$$
\eta_{m}=m \Delta \eta=\frac{2 \pi m}{\left(2 M^{\prime \prime}+1\right)}
$$

with $M^{\prime \prime}=\operatorname{Int}\left(\chi M^{\prime}\right)+1$ and $M^{\prime}=\operatorname{Int}\left(\chi^{\prime} W_{\eta}\right)+1$. Moreover,

$$
\begin{gathered}
D_{M^{\prime \prime}}(\eta)=\frac{\sin \left(\left(2 M^{\prime \prime}+1\right) \eta / 2\right)}{\left(2 M^{\prime \prime}+1\right) \sin (\eta / 2)}, \\
\Omega_{M}(\eta)=\frac{T_{M}\left[-1+2(\cos (\eta / 2) / \cos (\bar{\eta} / 2))^{2}\right]}{T_{M}\left[-1+2 / \cos ^{2}(\bar{\eta} / 2)\right]}
\end{gathered}
$$


are the Dirichlet and Tschebyscheff sampling functions [62], wherein $T_{M}(\eta)$ is the Tschebyscheff polynomial of degree $M=M^{\prime \prime}-M^{\prime}$ and $\bar{\eta}=p \Delta \eta$.

It is worth noting that small variations of $\eta$ correspond to very large changes of $\phi$ in the neighbourhood of the poles $(\vartheta=0$ and $\vartheta=\pi)$, so that, when interpolating the voltage in these zones, the enlargement bandwidth factor $\chi^{\prime}$ must be properly increased in order to avoid a significant growth of the bandlimitation error.

The OSI expansion (26) can be employed to determine the "intermediate samples," that is, the reduced voltage values at the intersection points between the spiral and the meridian curve passing through the observation point $P$. Once these samples have been evaluated, the voltage at the point $P$ can be reconstructed by means of the following OSI expansion:

$$
\begin{aligned}
& V(\xi(\vartheta), \varphi) \\
& \quad=e^{-j \gamma(\xi)} \sum_{n=n_{0}-q+1}^{n_{0}+q} \widetilde{V}\left(\xi_{n}\right) \Omega_{N}\left(\xi-\xi_{n}\right) D_{N^{\prime \prime}}\left(\xi-\xi_{n}\right),
\end{aligned}
$$

where $2 q$ is the number of retained intermediate samples $\widetilde{V}\left(\xi_{n}\right), n_{0}=\operatorname{Int}\left[\left(\xi-\xi_{0}\right) / \Delta \xi\right]$ is the index of the sample nearest (on the left) to the point $P, N=N^{\prime \prime}-N^{\prime}$,

$$
\xi_{n}=\xi_{n}(\varphi)=k \varphi+n \Delta \xi=\xi_{0}+n \Delta \xi,
$$

and the other symbols have the same or analogous meanings as in (26).

The effectiveness of the OSI algorithms depends on the choice of $\chi^{\prime}, \chi$, and $p, q$. Values of $\chi^{\prime}$ slightly greater than unity are enough to ensure small aliasing errors in the case of electrically large antennas [30]. A rigorous procedure to properly select it is described in [62]. As regards the choice of the parameters controlling the truncation error, that is, the oversampling factor $\chi$ and the numbers $p, q$ of the retained samples, it can be numerically made as in [31]. Note that such an error can be decreased on increasing the parameters $p, q$ and/or $\chi$. An increase of $p, q$ implies a growth of the interpolation time, whereas an increase of $\chi$ reflects in a growth of needed NF data and the related measurement time. In practice, the choice of these parameters is done in such a way that the error due to the interpolation (aliasing plus truncation) must be smaller than the measurement one.

\section{Numerical and Experimental Results}

Some representative numerical and experimental results relevant to the spherical spiral scanning case when considering quasi-planar and electrically long antennas, respectively, are reported in the following for reader's convenience. The interested reader can find other numerical and experimental results relevant to the spherical spiral scanning case as well as to the helicoidal and the planar spiral one in the quoted references. The reason for the choice of spherical spiral scanning case, notwithstanding its less effectiveness from the data reduction and measurement time saving viewpoints with respect to the helicoidal and planar spiral scan, is due to the fact that such a scanning, as the spherical one,

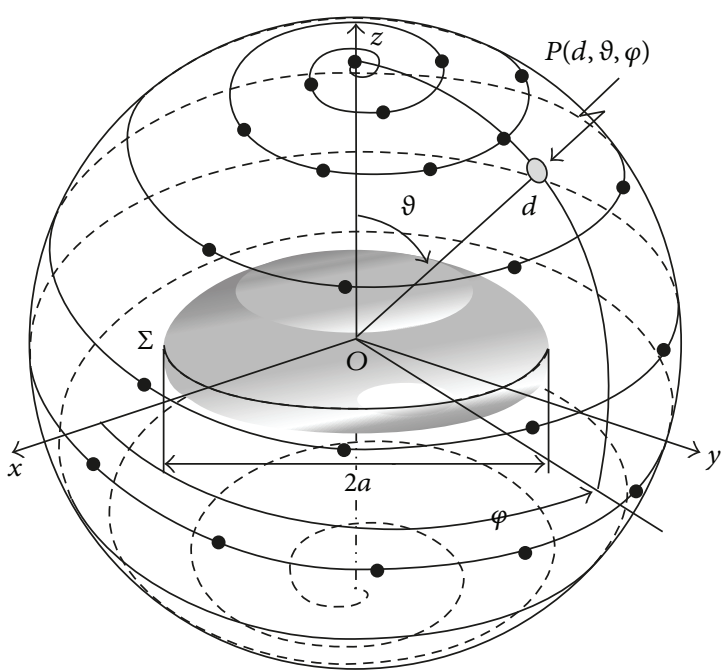

FIGURE 7: Spherical spiral scanning for a quasi-planar antenna.

allows the reconstruction of the whole AUT radiation pattern. Moreover, since it allows the characterization of planar, quasi-planar and elongated antennas, commonly adopted in modern wireless communication systems, the flexible AUT modellings adopted in the following examples have been employed also in the planar spiral scanning and in the helicoidal one, respectively. On the other hand, these two AUT modellings together with the already described ellipsoidal ones provide a whole survey on how to effectively model nonspherical antennas.

The following numerical simulations are relevant to the spherical spiral scanning and to a quasi-planar antenna modelled as enclosed in a double bowl, that is, a surface $\Sigma$ formed by two circular "bowls" [48] with the same aperture diameter $2 a$, but with bending radii $c$ and $c^{\prime}$ of the upper and lower arcs eventually different to fit better the actual AUT geometry (see Figure 7). It can be easily recognized that the surface $\Sigma$ coincides with a sphere if $c=c^{\prime}=a$, it becomes a half-sphere if $c=0$ and $c^{\prime}=a$, and it reduces to a circular dish for $c=c^{\prime}=0$. The expressions of the bandwidth $W_{\xi}=$ $\beta \ell^{\prime} / 2 \pi$ and of the parameterization $\xi$ relevant to a meridian and the related phase function $\gamma$ (see (8)) can be obtained by properly taking into account the geometry of $\Sigma$. It can be easily verified that the expressions of $R_{1,2}$ and $s_{1,2}^{\prime}$ change depending on the location of the tangent points $P_{1,2}$ and that five cases must be considered for $\vartheta$ ranging in $[0, \pi]$. Their explicit expressions can be found in [48]. The reported tests refer to a spiral lying on sphere of radius $d=30 \lambda$ and to three uniform planar circular arrays placed at $z=-5 \lambda, 0 \lambda, 5 \lambda$, having radius equal to $15 \lambda, 20 \lambda$, and $15 \lambda$, respectively. Their elements are elementary Huygens sources linearly polarized along $y$ and are radially and azimuthally spaced by $0.5 \lambda$. Such an antenna has been fitted by a double bowl with $c=c^{\prime}=5.3 \lambda$ and $a=20 \lambda$, and an open-ended circular waveguide, having radius $\rho^{\prime}=0.338 \lambda$, has been considered as probe.

The reconstructions of the amplitude and phase of the probe voltage $V$ on the meridian at $\varphi=0^{\circ}$ are reported in Figures 8 and 9, respectively. In both cases, a very good 


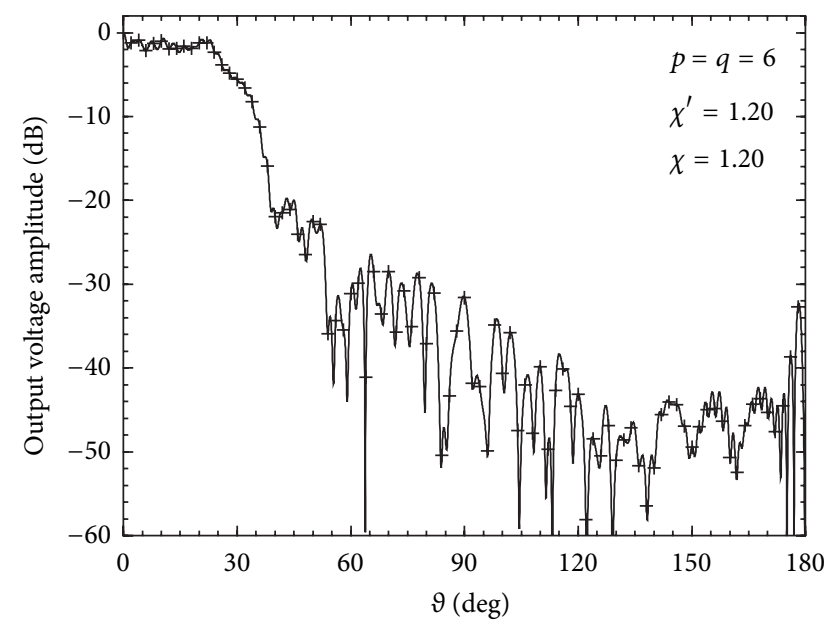

Figure 8: Amplitude of the probe voltage $V$ on the meridian at $\varphi=$ $0^{\circ}$. Solid line: exact. Crosses: interpolated.

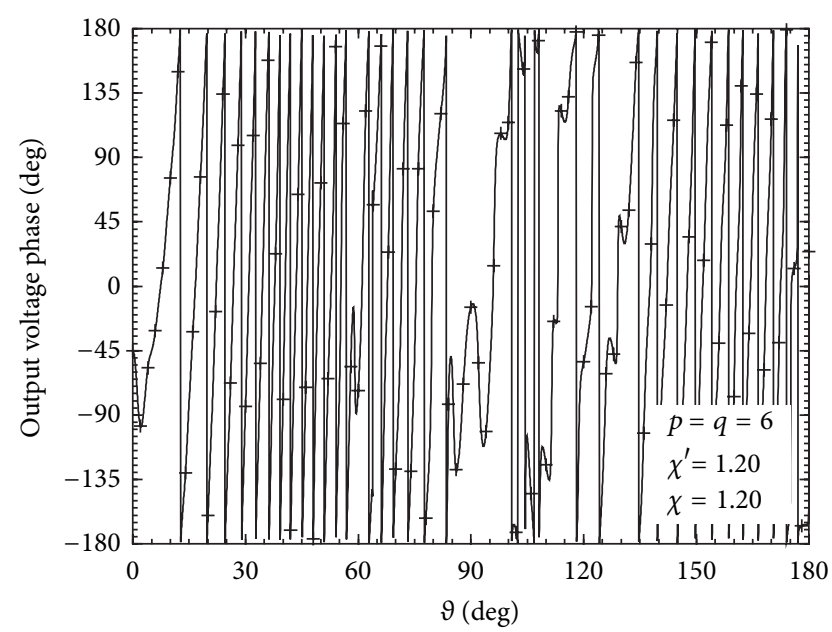

Figure 9: Phase of the probe voltage $V$ on the meridian at $\varphi=0^{\circ}$. Solid line: exact. Crosses: interpolated.

agreement between the exact and the reconstructed plot results. Note that an enlargement bandwidth factor such that the sample spacing is reduced by a factor 11 has been adopted in the zones of the spiral determined by the 46 samples around the poles. The accuracy in the NF interpolation is also confirmed by the values of the maximum and meansquare errors (normalized to the voltage maximum value on the sphere) relevant to the probe voltage $V$ shown in Figures 10 and 11 for $p=q$ ranging from 2 to $10, \chi^{\prime}=1.20$ (save for the polar zones), and $\chi=1.10,1.15,1.20,1.25$. As expected, they decrease to very low values on increasing the oversampling factor and/or the number of retained samples. Note that, according to these plots, the maximum and meansquare reconstruction errors corresponding to the chosen values of the OSI parameters are about $-45 \mathrm{~dB}$ and $-60 \mathrm{~dB}$, respectively. At last, the developed interpolation algorithm has been applied to retrieve the NF data needed to carry out the spherical NF-FF transformation [55], as modified

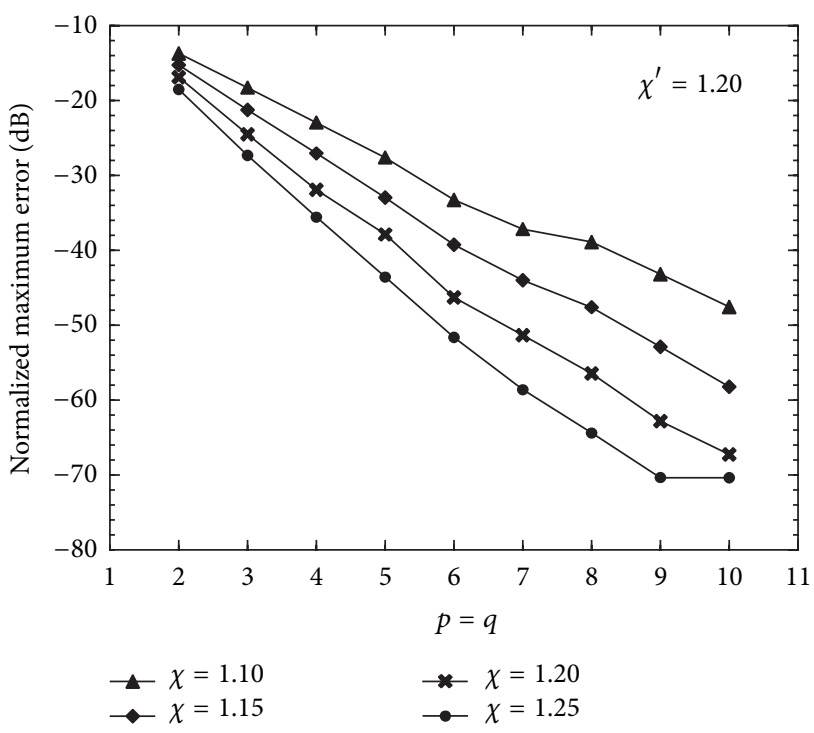

FIGURE 10: Normalized maximum errors in the reconstruction of $V$.

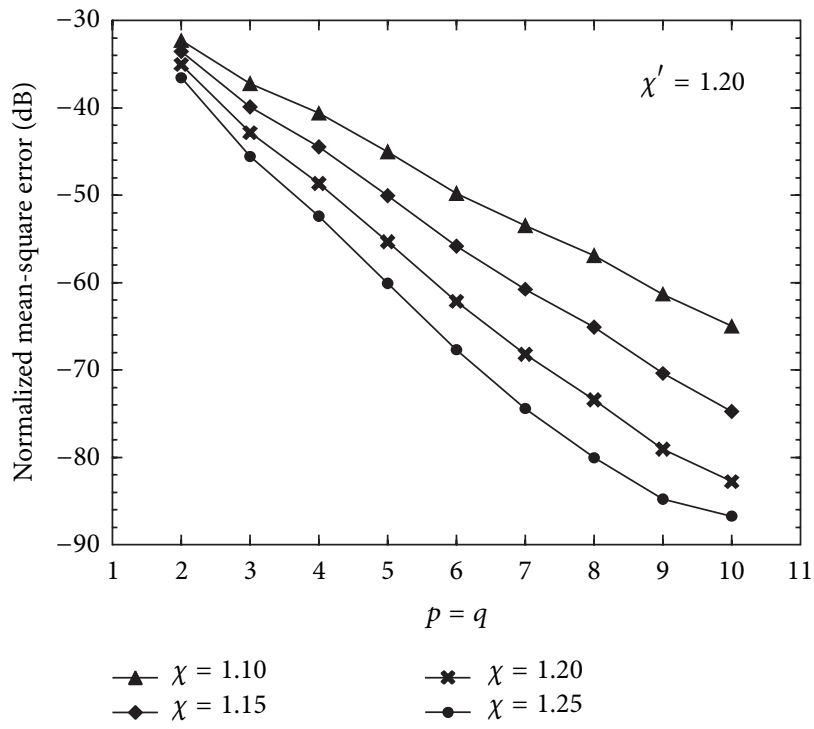

FIGURE 11: Normalized mean-square errors in the reconstruction of $V$.

in $[63,64]$. The reconstructed FF pattern in the H-plane is shown in Figure 12. As can be seen, the reconstruction is very accurate, thus assessing the overall effectiveness of the technique.

It is worthy to note that the number of samples on the spiral is 28483, significantly less than the one (43664) needed by the approach in [37]. In particular, the number of "regular samples" at spacing $\Delta \eta$ is 27563, whereas the number of "extra samples" at reduced spacing is 920. Moreover, the number of samples collected along the spiral is much less than that (130562) required by the NF-FF transformation with spherical scanning [55].

For reader's convenience, we report in the following some experimental results already published in [51] and 


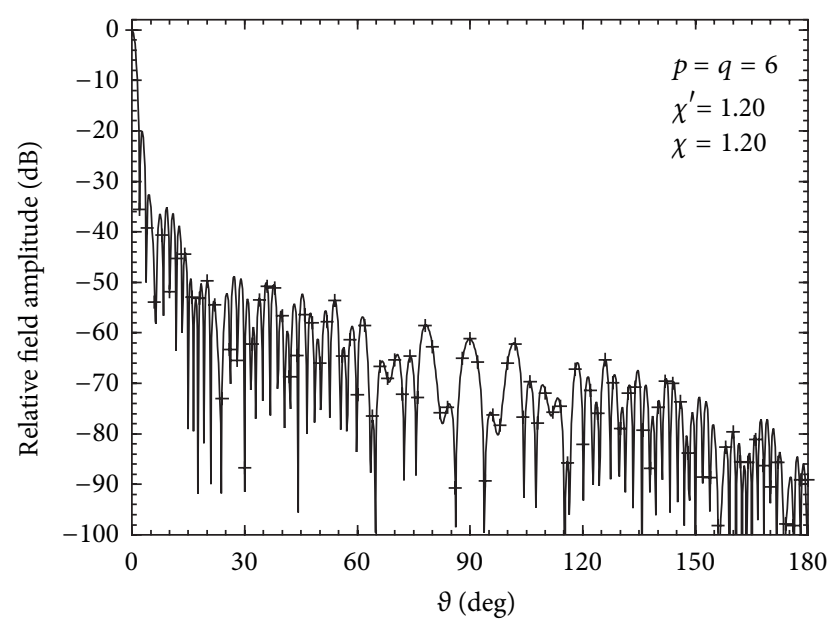

FIGURE 12: H-plane pattern. Solid line: exact. Crosses: reconstructed from NF measurements.

relevant to the spherical spiral scanning when dealing with an electrically long antenna modelled as enclosed in a rounded cylinder, namely, a cylinder of height $h^{\prime}$ ended in two halfspheres of radius $a^{\prime}$ (see Figure 13). Also such a modelling is quite general, containing the spherical one as particular case $\left(h^{\prime}=0\right)$, and allows fitting very well many real antennas [1-19] by properly choosing the values of the parameters $h^{\prime}$ and $a^{\prime}$. The expressions of the bandwidth $W_{\xi}=\beta \ell^{\prime} / 2 \pi$ and of $\xi$ and $\gamma$ (see (8)) can be again easily obtained by taking into account the geometry of $\Sigma$. As before, the expressions of $R_{1,2}$ and $s_{1,2}^{\prime}$ change depending on the location of the tangency points $P_{1,2}$, but now three cases must be considered when $\vartheta$ varies in the range $[0, \pi]$. Their explicit expressions can be found in [51]. The reported experimental results have been carried out in the anechoic chamber of the Antenna Characterization Lab of University of Salerno, which is provided with a roll ( $\varphi$ axis) over azimuth ( $\vartheta$ axis) spherical NF facility supplied by MI Technologies. The chamber is covered with pyramidal absorbers ensuring a wall reflectivity less than $-40 \mathrm{~dB}$. An open-ended WR90 rectangular waveguide is used as probe and its response has been collected on a spiral wrapping a sphere having radius $d=45.2 \mathrm{~cm}$. The amplitude and phase measurements are accomplished by means of a vector network analyzer. The considered AUT is a $\mathrm{X}$-band resonant slotted waveguide array made by PROCOM $\mathrm{A} / \mathrm{S}$ and working at $10.4 \mathrm{GHz}$, realized by cutting 12 roundended slots on both the broad walls of a WR90 rectangular waveguide and soldering two cylinders on its narrow walls (see Figure 14). According to the adopted representation, such an antenna has been modelled by a rounded cylinder with $h^{\prime}=28.28 \mathrm{~cm}$ and $a^{\prime}=2.60 \mathrm{~cm}$ and mounted in such a way that the broad walls are parallel to the plane $y=0$ and its axis is coincident with the $z$ one.

The effectiveness of the two-dimensional OSI expansion is assessed by comparing the amplitude and phase of the recovered voltage $V$ relevant to the meridian $\varphi=0^{\circ}$ with those directly acquired on the same meridian (see Figures 15 and 16). As it can be seen, there is a very good agreement

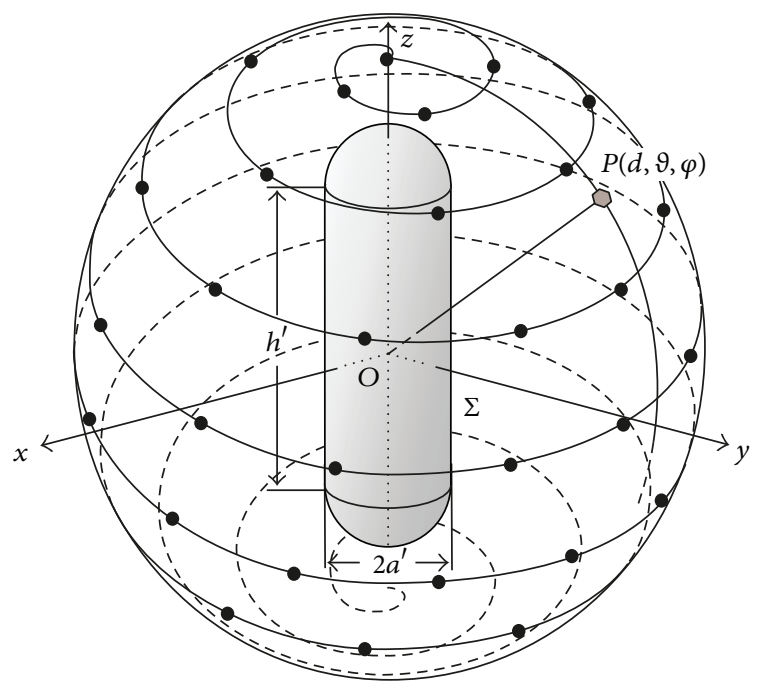

Figure 13: Spherical spiral scanning for an elongated antenna.

between the reconstructed voltage and the directly measured one, save for the zones characterized by a very low level, wherein the error is due to the noise and the residual reflections from the anechoic chamber walls. It is useful to note that an enlargement bandwidth factor $\chi^{\prime}$ such that the spacing among the samples is reduced exactly by a factor 5 has been adopted in the zones of the spiral determined by the 20 samples around each pole.

At last, the E-plane and H-plane FF patterns reconstructed from the NF set of measurements acquired by means of the spherical spiral scan are compared in Figures 17 and 18 with those (references) obtained from the NF data directly measured on the classical spherical grid. In both cases, the software package MI-3000, which implements the classical spherical NF-FF transformation [55], has been used to get the FF patterns. In particular, the reconstruction error, useful to appreciate the error levels, is also reported in Figure 18. As can be seen, the reconstructions are very accurate, thus fully confirming also from the experimental viewpoint the effectiveness of the described technique.

Note that the number of used samples is 1024, significantly less than those (3622 and 5100) required by the NF-FF transformation with spiral scanning [37] based on the spherical AUT modelling and by the MI Technologies software package implementing the classical NF-FF transformation [55], respectively. In particular, the number of regular samples is 864 , whereas the number of extra samples at reduced spacing is 160 .

It is worth noting that, in this example, the reduction rates of the needed NF data with respect to those required in the spherical spiral scan [37] using the spherical modelling and in the classical one are much greater than the corresponding ones obtained in the former example. This is a general validity result: for long antennas, usually adopted in urban radio base station [7-9], the time saving achievable by using the appropriate spiral scanning technique is usually remarkably greater than that for the quasi-planar ones, since the number of spiral turns is related to the length of $C^{\prime}$, whereas the 


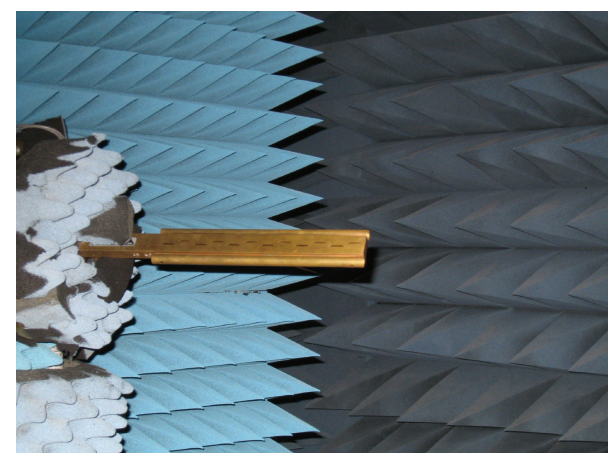

FIgUre 14: Photo of the X-band resonant slotted waveguide array.

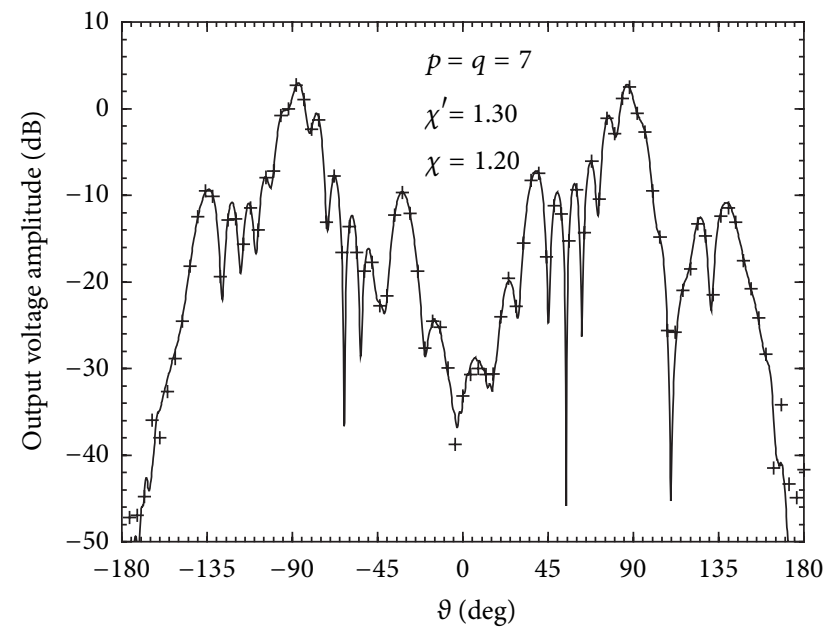

Figure 15: Amplitude of $V$ on the meridian at $\varphi=0^{\circ}$. Solid line: measured. Crosses: recovered from NF data acquired via the spherical spiral scanning.

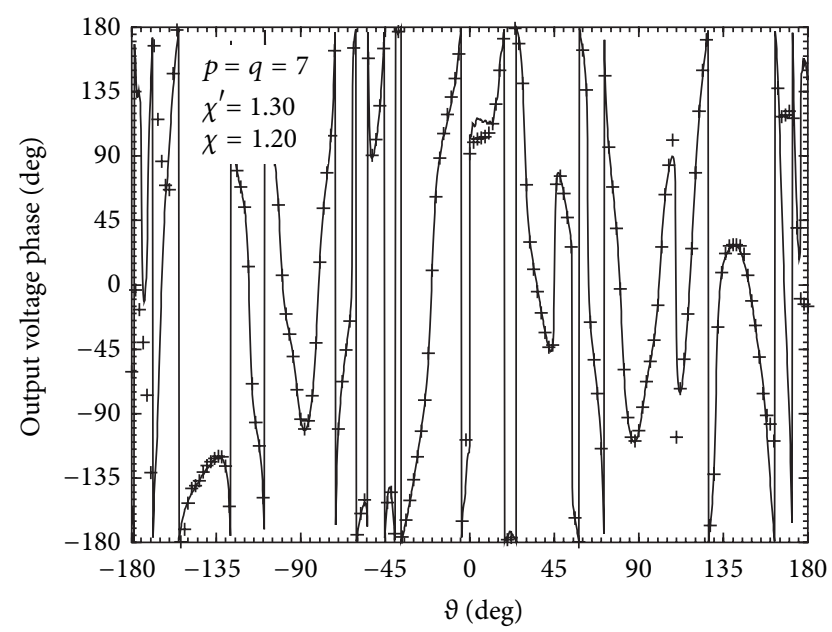

Figure 16: Phase of $V$ on the meridian at $\varphi=0^{\circ}$. Solid line: measured. Crosses: recovered from NF data acquired via the spherical spiral scanning.

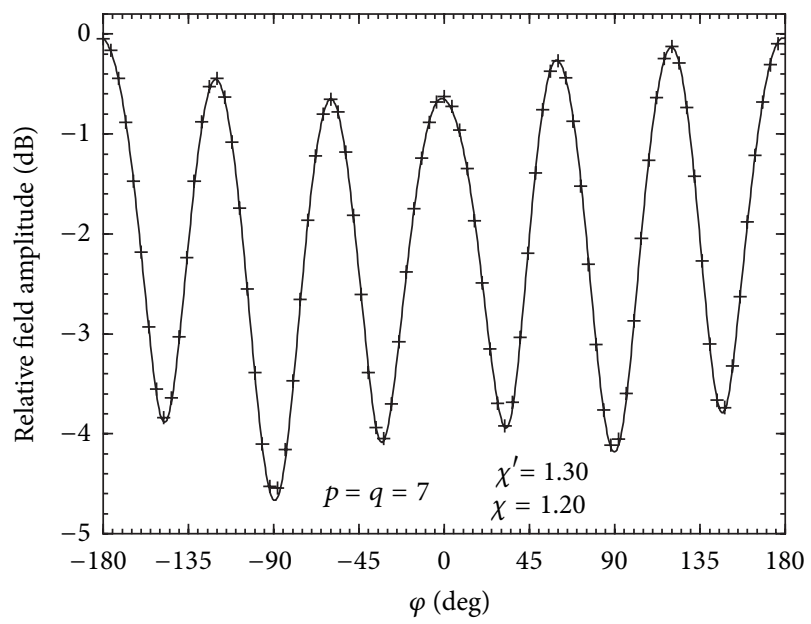

FIGURE 17: E-plane pattern. Solid line: reference. Crosses: recovered from NF data acquired via the spherical spiral scanning.

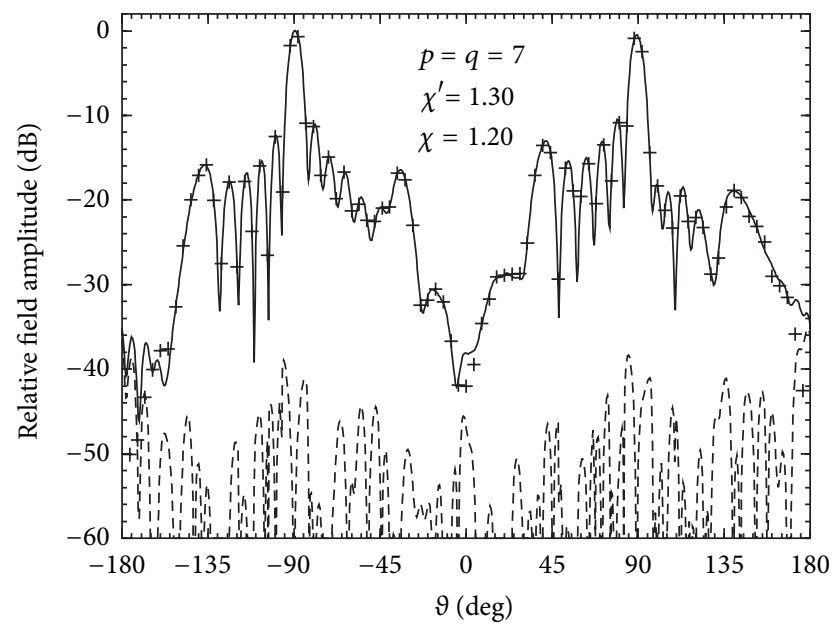

FIGURE 18: H-plane pattern. Solid line: reference. Crosses: recovered from NF data acquired via the spherical spiral scan. Dashed line: reconstruction error.

average number of samples on each turn depends on the maximum transverse radius of $\Sigma$.

\section{Conclusions}

This paper provides a comprehensive overview of the nonredundant NF-FF transformations using fast spiral scanning techniques, which allow a drastic time saving, since they are realized through continuous and synchronized movements of the positioning systems and require a reduced number of NF measurements. The authors have first recalled the results on the nonredundant representations of EM fields, which constitute the theoretical background of the spiral scanning techniques. Then, they have described the reasoning involved in the heuristic approach that allowed the development of the unified theory concerning the spiral scannings for nonspherical antennas from that for the quasi-spherical ones. The OSI algorithm, used to determine the voltage measured 
by a nondirective probe on a quite arbitrary rotational surface from a nonredundant number of its samples collected along a proper spiral, has been also presented. Although this theory is valid for spirals lying on quite arbitrary rotational surfaces, its application to spirals wrapping the conventional scanning surfaces has made possible the development of accurate NF-FF transformations which use a nonredundant number of NF spiral data and allow remarkably reducing the measurement time, since the NF data needed by the corresponding classical NF-FF transformation are accurately reconstructed from those collected on the spiral path. NFFF transformation techniques allowing a remarkable time saving without losing the efficiency of the classical ones are so made available to the antenna designers and to the measurement community involved in the development of the modern wireless communication systems.

\section{Conflict of Interests}

The authors declare that there is no conflict of interests regarding the publication of this paper.

\section{References}

[1] B. Allen, M. Dohler, E. E. Okon, W. Q. Malik, A. K. Brown, and D. J. Edwards, Ultra-Wideband Antennas and Propagation for Communications, Radar and Imaging, Wiley, New York, NY, USA, 2007.

[2] T. Karacolak and E. Topsakal, "A double-sided rounded bow-tie antenna (DSRBA) for UWB communication," IEEE Antennas and Wireless Propagation Letters, vol. 5, no. 1, pp. 446-449, 2006.

[3] D. Caratelli, R. Cicchetti, G. Bit-Babik, and A. Faraone, "A perturbed E-shaped patch antenna for wideband WLAN applications," IEEE Transactions on Antennas and Propagation, vol. 54, no. 6, pp. 1871-1874, 2006.

[4] G. Cappelletti, D. Caratelli, R. Cicchetti, and M. Simeoni, "A low-profile printed drop-shaped dipole antenna for wideband wireless applications," IEEE Transactions on Antennas and Propagation, vol. 59, no. 10, pp. 3526-3535, 2011.

[5] Y. Lim, Y. J. Yoon, and B. Jung, "Parasitic-element-loaded UWB antenna with band-stop function for mobile handset wireless USB," International Journal of Antennas and Propagation, vol. 2012, Article ID 427841, 6 pages, 2012.

[6] J. Wu, Z. Zhao, Z. Nie, and Q. Liu, "A compact printed dipole antenna for wideband wireless applications," Progress In Electromagnetics Research C, vol. 50, pp. 95-102, 2014.

[7] A. Faraone, R. Y.-S. Tay, K. H. Joyner, and Q. Balzano, "Estimation of the average power density in the vicinity of cellular base-station collinear array antennas," IEEE Transactions on Vehicular Technology, vol. 49, no. 3, pp. 984-996, 2000.

[8] S. Blanch, J. Romeu, and A. Cardama, "Near field in the vicinity of wireless base-station antennas: an exposure compliance approach," IEEE Transactions on Antennas and Propagation, vol. 50, no. 5, pp. 685-692, 2002.

[9] R. Cicchetti, A. Faraone, and Q. Balzano, "A uniform asymptotic evaluation of the field radiated from collinear array antennas," IEEE Transactions on Antennas and Propagation, vol. 51, no. 1, pp. 89-102, 2003.

[10] A. Falahati, M. Naghshvarianjahromi, and R. M. Edwards, "Wideband fan-beam low-sidelobe array antenna using grounded reflector for DECT, 3G, and ultra-wideband wireless applications," IEEE Transactions on Antennas and Propagation, vol. 61, no. 2, pp. 700-706, 2013.

[11] D. Caratelli, M. C. Viganó, G. Toso, P. Angeletti, A. Shibelgut, and R. Cicchetti, "A hybrid deterministic/metaheuristic synthesis technique for non-uniformly spaced linear printed antenna arrays," Progress in Electromagnetics Research, vol. 142, pp. 107121, 2013.

[12] Y. M. M. Antar, "Antennas for wireless communication: recent advances using dielectric resonators," IET Circuits, Devices and Systems, vol. 2, no. 1, pp. 133-138, 2008.

[13] M. Simeoni, R. Cicchetti, A. Yarovoy, and D. Caratelli, "Plasticbased supershaped dielectric resonator antennas for wide-band applications," IEEE Transactions on Antennas and Propagation, vol. 59, no. 12, pp. 4820-4825, 2011.

[14] K. W. Leung, E. H. Lim, and X. S. Fang, "Dielectric resonator antennas: from the basic to the aesthetic," Proceedings of the IEEE, vol. 100, no. 7, pp. 2181-2193, 2012.

[15] M. Ali, S. S. Stuchly, and K. Caputa, "A compact flat reflector antenna for potential base station applications," Microwave and Optical Technology Letters, vol. 18, no. 5, pp. 319-320, 1998.

[16] W. H. Theunissen and W. D. Burnside, "Contoured beam reflector antenna for wireless applications," IEEE Transactions on Antennas and Propagation, vol. 50, no. 2, pp. 205-210, 2002.

[17] I. B. Trad, J. M. Floc'h, H. Rmili, M. Drissi, and F. Choubani, "A planar reconfigurable radiation pattern dipole antenna with reflectors and directors for wireless communication applications," International Journal of Antennas and Propagation, vol. 2014, Article ID 593259, 10 pages, 2014.

[18] D. Caratelli and M. C. Viganó, "Analytical synthesis technique for linear uniform-amplitude sparse arrays," Radio Science, vol. 46, no. 4, Article ID RS4001, pp. 1-6, 2011.

[19] D. Caratelli and M. C. Viganó, "A novel deterministic synthesis technique for constrained sparse array design problems," IEEE Transactions on Antennas and Propagation, vol. 59, no. 11, pp. 4085-4093, 2011.

[20] R. C. Johnson, H. A. Ecker, and J. S. Hollis, "Determination of far-field antenna patterns from near-field measurements," Proceedings of the IEEE, vol. 61, no. 12, pp. 1668-1694, 1973.

[21] A. D. Yaghjian, "An overview of near-field antenna measurements," IEEE Transactions on Antennas and Propagation, vol. 34, no. 1, pp. 30-45, 1986.

[22] J. Appel-Hansen, J. D. Dyson, E. S. Gillespie, and T. G. Hickman, "Antenna measurements," in The Handbook of Antenna Design, K. Milne, A. D. Olver, and P. Knight, Eds., chapter 8, Peter Peregrinus, London, UK, 1986.

[23] E. S. Gillespie, "Special Issue on near-field scanning techniques," IEEE Transactions on Antennas and Propagation, vol. 36, no. 6, pp. 727-901, 1988.

[24] D. Slater, Near-Field Antenna Measurements, Artech House, Boston, Mass, USA, 1991.

[25] S. F. Gregson, J. McCormick, and C. G. Parini, Principles of Planar Near-Field Antenna Measurements, The Institution of Engineering and Technology, London, UK, 2007.

[26] M. H. Francis and R. W. Wittmann, "Near-field scanning measurements: theory and practice," in Modern Antenna Handbook, C. A. Balanis, Ed., John Wiley \& Sons, Hoboken, NJ, USA, 2008.

[27] M. H. Francis, Ed., IEEE Recommended Practice for Near-Field Antenna Measurements, IEEE Standard 1720, 2012.

[28] C. Gennarelli, A. Capozzoli, L. J. Foged, J. Fordham, and D. J. van Rensburg, "Recent advances in near-field to far-field 
transformation techniques," International Journal of Antennas and Propagation, vol. 2012, Article ID 243203, 3 pages, 2012.

[29] R. G. Yaccarino, Y. Rahmat-Samii, and L. I. Williams, "Bi-polar planar near-field measurement technique, part II: near-field to far-field transformation and holographic imaging methods," IEEE Transactions on Antennas and Propagation, vol. 42, no. 2, pp. 196-204, 1994.

[30] O. M. Bucci and G. Franceschetti, "On the spatial bandwidth of scattered fields," IEEE Transactions on Antennas and Propagation, vol. 35, no. 12, pp. 1445-1455, 1987.

[31] O. M. Bucci, C. Gennarelli, and C. Savarese, "Representation of electromagnetic fields over arbitrary surfaces by a finite and nonredundant number of samples," IEEE Transactions on Antennas and Propagation, vol. 46, no. 3, pp. 351-359, 1998.

[32] O. M. Bucci and C. Gennarelli, "Application of nonredundant sampling representations of electromagnetic fields to NF-FF transformation techniques," International Journal of Antennas and Propagation, vol. 2012, Article ID 319856, 14 pages, 2012.

[33] F. D’Agostino, F. Ferrara, C. Gennarelli, R. Guerriero, M. Migliozzi, and G. Riccio, Near-Field-Far-Field Transformation Techniques with Spiral Scan Nings, CUES, Salerno, Italy, 2009.

[34] J. C. Bolomey, B. J. Cown, G. Fine et al., "Rapid near-field antenna testing via arrays of modulated scattering probes," IEEE Transactions on Antennas and Propagation, vol. 36, no. 6, pp. 804-814, 1988.

[35] R. G. Yaccarino and L. I. Williams, "Linear spiral sampling for the bipolar planar near-field antenna measurement technique," IEEE Transactions on Antennas and Propagation, vol. 44, no. 7, pp. 1049-1051, 1996.

[36] O. M. Bucci, C. Gennarelli, G. Riccio, and C. Savarese, "Nonredundant NF-FF transformation with helicoidal scanning," Journal of Electromagnetic Waves and Applications, vol. 15, no. 11, pp. 1507-1519, 2001.

[37] F. D’Agostino, C. Gennarelli, G. Riccio, and C. Savarese, “Theoretical foundations of near-field-far-field transformations with spiral scannings," Progress in Electromagnetics Research, vol. 61, pp. 193-214, 2006.

[38] F. D'Agostino, F. Ferrara, C. Gennarelli, R. Guerriero, and M. Migliozzi, "Experimental results validating the near-field to far-field transformation technique with helicoidal scan," Open Electrical and Electronic Engineering Journal, vol. 4, pp. 10-15, 2010.

[39] F. D’Agostino, F. Ferrara, C. Gennarelli, R. Guerriero, and M. Migliozzi, "Near-field-far-field transformation technique with helicoidal scanning for elongated antennas," Progress in Electromagnetics Research B, vol. 4, pp. 249-261, 2008.

[40] F. D’Agostino, F. Ferrara, C. Gennarelli, R. Guerriero, and M. Migliozzi, "Laboratory tests assessing the effectiveness of the NF-FF transformation with helicoidal scanning for electrically long antennas," Progress in Electromagnetics Research, vol. 98, pp. 375-388, 2009.

[41] F. D’Agostino, F. Ferrara, J. A. Fordham et al., "An effective nearfield-far-field transformation technique for elongated antennas using a fast helicoidal scan," IEEE Antennas and Propagation Magazine, vol. 51, no. 4, pp. 134-141, 2009.

[42] F. D’Agostino, F. Ferrara, C. Gennarelli, R. Guerriero, and M. Migliozzi, "The unified theory of near-field-far-field transformations with spiral scannings for nonspherical antennas," Progress in Electromagnetics Research B, no. 14, pp. 449-477, 2009.

[43] O. M. Bucci, F. D’Agostino, C. Gennarelli, G. Riccio, and C. Savarese, "Probe compensated far-field reconstruction by near-field planar spiral scanning," IEE Proceedings: Microwaves, Antennas and Propagation, vol. 149, no. 2, pp. 119-123, 2002.

[44] F. D'Agostino, F. Ferrara, C. Gennarelli, R. Guerriero, and M. Migliozzi, "An effective NF-FF transformation technique with planar spiral scanning tailored for quasi-planar antennas," IEEE Transactions on Antennas and Propagation, vol. 56, no. 9, pp. 2981-2987, 2008.

[45] O. M. Bucci, F. D’Agostino, C. Gennarelli, G. Riccio, and C. Savarese, "Near-field-far-field transformation with spherical spiral scanning," IEEE Antennas and Wireless Propagation Letters, vol. 2, pp. 263-266, 2003.

[46] F. D’Agostino, F. Ferrara, J. A. Fordham, C. Gennarelli, R. Guerriero, and M. Migliozzi, "An experimental validation of the near-field-far-field transformation with spherical spiral scan," IEEE Antennas and Propagation Magazine, vol. 55, no. 3, pp. 228-235, 2013.

[47] F. DAgostino, F. Ferrara, C. Gennarelli, R. Guerriero, M. Migliozzi, and G. Riccio, "A nonredundant near-field to far-field transformation with spherical spiral scanning for nonspherical antennas," The Open Electrical Electronic Engineering Journal, vol. 3, pp. 1-8, 2009.

[48] F. D’Agostino, F. Ferrara, C. Gennarelli, R. Guerriero, and M. Migliozzi, "Far-field reconstruction from a minimum number of spherical spiral data using effective antenna modelings," Progress In Electromagnetics Research B, no. 37, pp. 43-58, 2012.

[49] F. D’Agostino, F. Ferrara, C. Gennarelli, R. Guerriero, and M. Migliozzi, "Experimental assessment of an effective nearfield-far-field transformation with spherical spiral scanning for quasi-planar antennas," IEEE Antennas and Wireless Propagation Letters, vol. 12, pp. 670-673, 2013.

[50] F. D'Agostino, F. Ferrara, C. Gennarelli, R. Guerriero, and M. Migliozzi, "Far-field reconstruction from near-field data acquired via a fast spherical spiral scan: experimental evidences," Progress in Electromagnetics Research, vol. 140, pp. 719732, 2013

[51] F. D’Agostino, F. Ferrara, C. Gennarelli, R. Guerriero, and M. Migliozzi, "Experimental testing on an effective technique to reconstruct the far-field pattern of a long antenna from nearfield measurements acquired via spherical spiral scan," The Open Electrical Electronic Engineering Journal, vol. 8, pp. 1-9, 2014.

[52] F. D'Agostino, F. Ferrara, C. Gennarelli, R. Guerriero, and M. Migliozzi, "Measurements corner: efficient reconstruction of the pattern radiated by a long antenna from data acquired via a spherical-spiral-scanning near-field facility," IEEE Antennas and Propagation Magazine, vol. 56, no. 2, pp. 146-153, 2014.

[53] W. M. Leach Jr. and D. T. Paris, "Probe compensated near-field measurements on a cylinder," IEEE Transactions on Antennas and Propagation, vol. AP-21, no. 4, pp. 435-445, 1973.

[54] E. B. Joy, W. M. Leach Jr., G. P. Rodrigue, and D. T. Paris, "Applications of probe-compensated near-field measurements," IEEE Transactions on Antennas and Propagation, vol. 26, no. 3, pp. 379-389, 1978.

[55] J. Hald, J. E. Hansen, F. Jensen, and F. H. Larsen, Spherical NearField Antenna Measurement, IEE Electromagnetic Waves Series, Peter Peregrinus, London, UK, 1998, edited by J. E. Hansen.

[56] F. Dagostino, F. Ferrara, C. Gennarelli, R. Guerriero, and M. Migliozzi, "An innovative direct NF-FF transformation technique with helicoidal scanning," International Journal of Antennas and Propagation, vol. 2012, Article ID 912948, 9 pages, 2012. 
[57] F. D’Agostino, F. Ferrara, C. Gennarelli, R. Guerriero, and M. Migliozzi, "Evaluation of the far field radiated by long antennas directly from data acquired through a fast helicoidal scanning," Progress In Electromagnetics Research M, vol. 26, pp. 157-171, 2012.

[58] F. D’Agostino, F. Ferrara, C. Gennarelli, R. Guerriero, and M. Migliozzi, "An efficient transformation technique for the direct recovering of the antenna far-field pattern from near-field measurements collected along a helix," The Open Electrical \& Electronic Engineering Journal, vol. 7, no. 1, pp. 21-30, 2013.

[59] S. Costanzo and G. Di Massa, "Far-field reconstruction from phaseless near-field data on a cylindrical helix," Journal of Electromagnetic Waves and Applications, vol. 18, no. 8, pp. 10571071, 2004.

[60] S. Costanzo and G. Di Massa, "Near-field to far-field transformation with planar spiral scanning," Progress in Electromagnetics Research, vol. 73, pp. 49-59, 2007.

[61] O. M. Bucci, G. D’Elia, and M. D. Migliore, "Advanced field interpolation from plane-polar samples: experimental verification," IEEE Transactions on Antennas and Propagation, vol. 46, no. 2, pp. 204-210, 1998.

[62] O. M. Bucci, C. Gennarelli, and C. Savarese, "Optimal interpolation of radiated fields over a sphere," IEEE Transactions on Antennas and Propagation, vol. 39, no. 11, pp. 1633-1643, 1991.

[63] O. M. Bucci, F. D’Agostino, C. Gennarelli, G. Riccio, and C. Savarese, "Data reduction in the NF-FF transformation technique with spherical scanning," Journal of Electromagnetic Waves and Applications, vol. 15, no. 6, pp. 755-775, 2001.

[64] F. D’Agostino, F. Ferrara, C. Gennarelli, R. Guerriero, and M. Migliozzi, "Effective antenna modellings for NF-FF transformations with spherical scanning using the minimum number of data," International Journal of Antennas and Propagation, vol. 2011, Article ID 936781, 11 pages, 2011. 

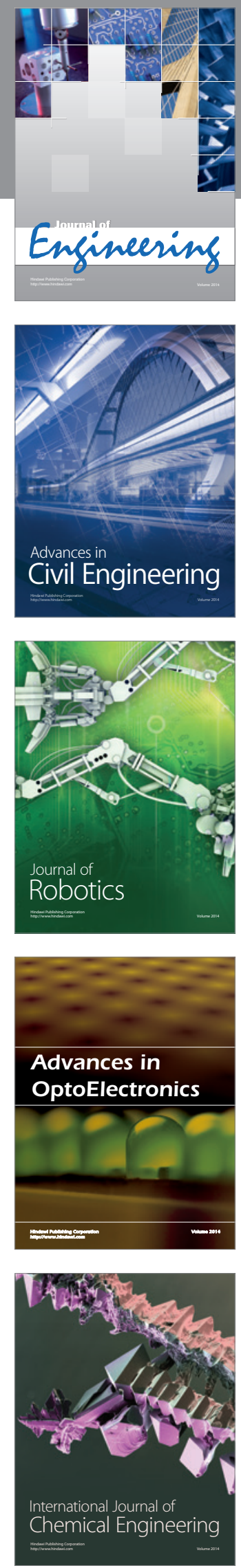

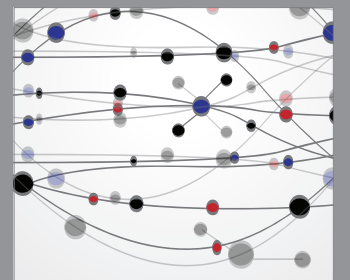

The Scientific World Journal
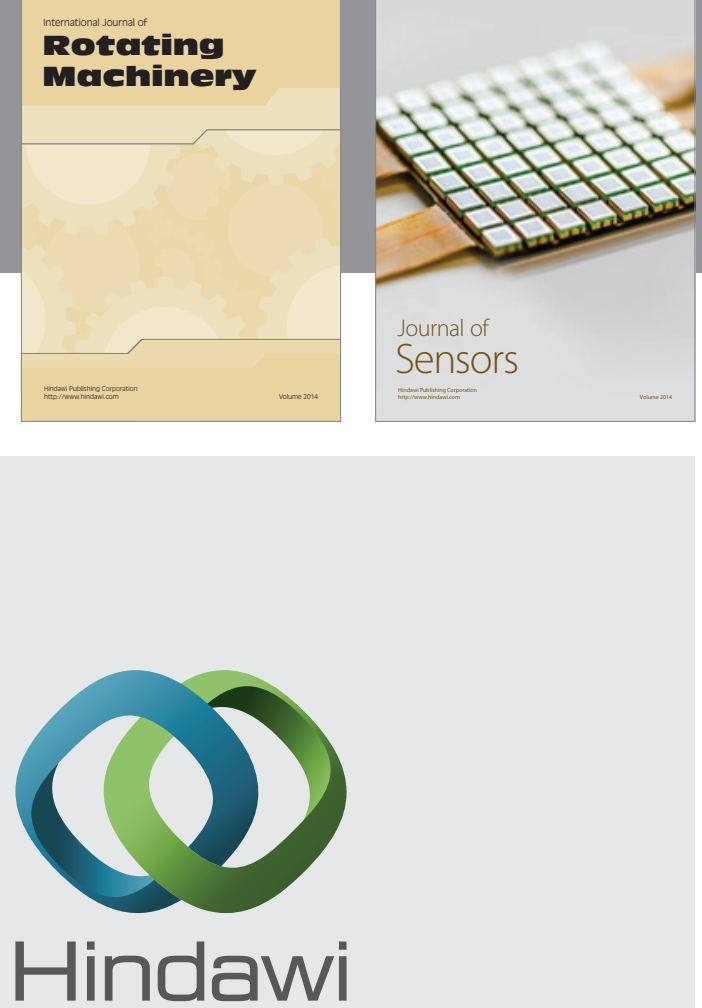

Submit your manuscripts at http://www.hindawi.com
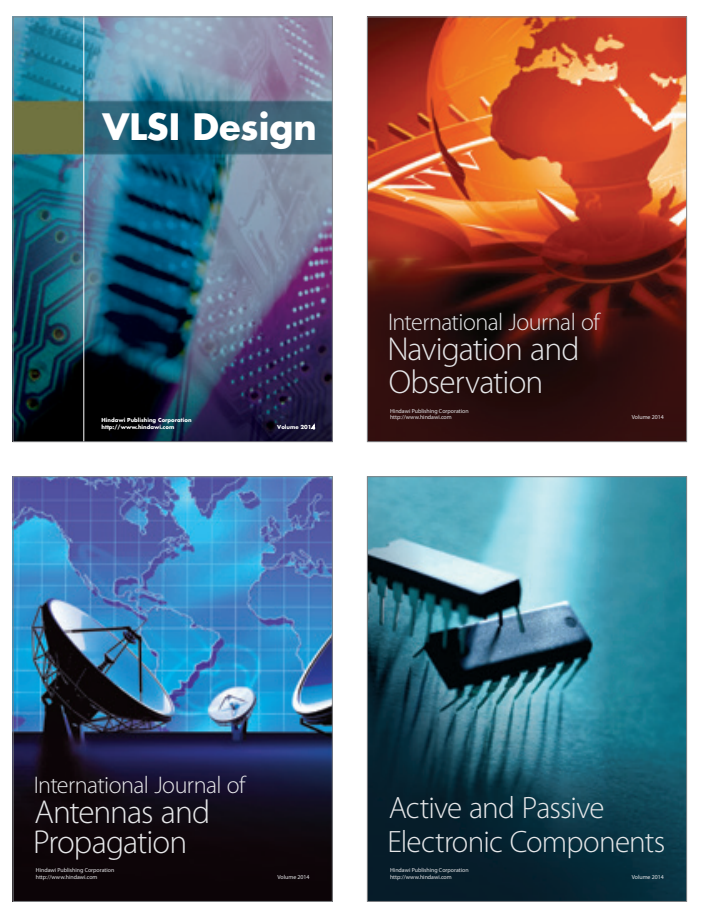
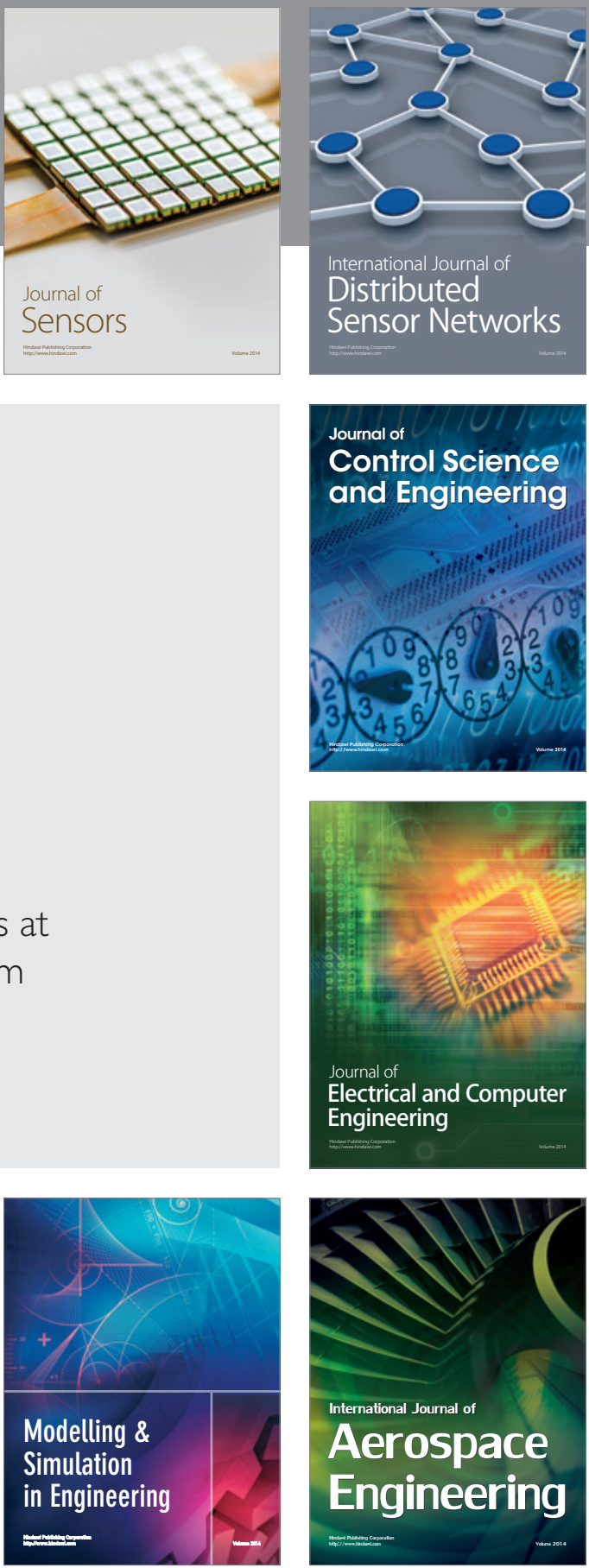

Journal of

Control Science

and Engineering
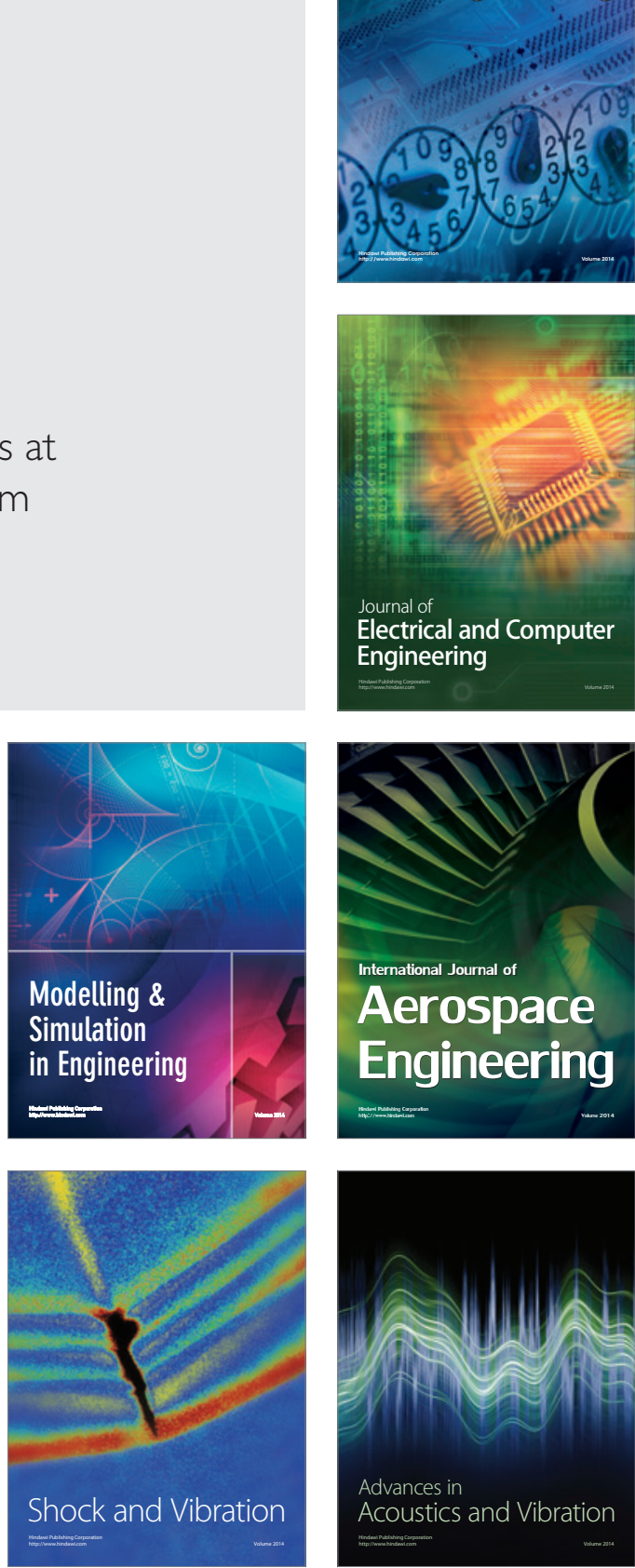